\title{
Isospin particle systems on quaternionic projective spaces
}

\author{
Stefano Bellucci $^{a}$, Sergey Krivonos ${ }^{b}$, Armen Nersessian ${ }^{c}$, Vahagn Yeghikyan $^{a, c}$ \\ ${ }^{a}$ INFN-Laboratori Nazionali di Frascati, Via E. Fermi 40, 00044, Frascati, Italy \\ ${ }^{b}$ Bogoliubov Laboratory of Theoretical Physics, JINR, 141980 Dubna, Russia \\ ${ }^{c}$ Yerevan State University, 1 Alex Manoogian St., Yerevan, 0025, Armenia
}

\begin{abstract}
We construct the isospin particle system on $n$-dimensional quaternionic projective spaces in the presence of BPST-instanton by the reduction from the free particle on $(2 n+1)$-dimensional complex projective space. Then we add to this system a "quaternionic oscillator potential" and show, that this oscillator-like system is superintegrable. We show, that besides the analogs of quadratic constants of motion of the spherical (Higgs) and $\mathbb{C} P^{n}$ - oscillators, it possesses the third-order constants of motion, which are functionally independent from the quadratic ones.
\end{abstract}

\section{Introduction}

Hopf maps play a distinguished role in theoretical physics, appearing, sometimes in a hidden way, in most of the key models. However, even the constructions, related with the second Hopf map, particularly, quaternionic projective spaces $\mathbb{H} P^{n}$, are not properly studied or/and used. In fact, explicitly quaternionic projective spaces appear in the construction of the multi-instanton of self-dual Yang-Mills theory only [1. Even the classical and quantum mechanical systems on quaternionic projective spaces (except systems on $\mathbb{H} P^{1}$ i.e. the four-dimensional sphere) were not paid enough attention. On the other hand, there is no doubt, that on these spaces one can easily construct the integrable systems of isospin particles interacting with instantons: due to the existence of the well-known fibration $S^{2} \rightarrow \mathbb{C} P^{2 n+1} \rightarrow \mathbb{H} P^{n}$, the inclusion of instanton fields should not destroy the symmetries of the $S p(n)$-invariant systems on $\mathbb{H} P^{n}$. This is similar to the well-known preservation of the symmetries of $U(N)$ invariant systems on complex projective spaces after inclusion of the constant magnetic field, which reflects the existence of the fibration $S^{1} \rightarrow S^{2 n+1} \rightarrow \mathbb{C} P^{n}$ related with the first Hopf map. Moreover, it is obvious, that on $\mathbb{H} P^{n}$ one can define the $s p(n+1)$-invariant "quaternionic Landau problem", i.e. a free particle interacting with a constant (BPST) instanton field, which is the quaternionic analog of the "Landau problem" on $\mathbb{C} P^{n}$ : a $s u(n+1)$ invariant system of particles interacting with a constant magnetic field. The simplest, one-dimensional quaternionic Landau problem on $\mathbb{H} P^{1}=S^{4}$ [2 has been used previously for developing the model of the "four-dimensional Hall effect" [3, and, by this reason it attracted much attention (see, e.g. [4] and the brief review [5]). Nevertheless, all these studies were restricted to the systems on $\mathbb{H} P^{1}=S^{4}$, and there was no attempts to consider even the higher-dimensional quaternionic Landau problem. Though, technically this should not be a difficult problem, since the fibration $S^{2} \rightarrow \mathbb{C} P^{2 n+1} \rightarrow \mathbb{H} P^{n}$ allows one to construct the lift (or reformulate it) from the free particle systems on the complex projective space $\mathbb{C} P^{2 n+1}$. For the $n=1$ case this fibration was widely explored in the study of the four-dimensional Hall effect, while the $S^{4}$-Landau problem in itself was explicitly constructed by the Hamiltonian reduction of the free particle on $\mathbb{C} P^{3}$ in [6]. Below we will fill the mentioned gap, presenting the detail description of the Hamiltonian reduction of the free particle on $\mathbb{C} P^{2 n+1}$ to the quaternionic Landau problem on $\mathbb{H} P^{n}$ (see the Third Section). Besides, we will present the superintegrable analog of the oscillator on quaternionic projective spaces, which respects the inclusion of a constant instanton field. In contrast with spherical (Higgs) 9] and $\mathbb{C} P^{n}$ [7 oscillators, whose hidden symmetries are of the second order in momenta, our model has additional constants of motion, which are of the third order in momenta(see the Fourth Section). In the Second Section we describe the fibration $S^{2} \rightarrow \mathbb{C} P^{2 n+1} \rightarrow \mathbb{H} P^{n}$.

\section{$2 \quad \mathbb{C} P^{2 n+1} \rightarrow \mathbb{H} P^{n}$ fibration}

In this Section we formulate the fibration and define the mathematical objects we are going to deal with.

First, let us notice, that the definition of projective spaces define an infinite series of fibrations, the natural projections of which are called Hopf maps. Indeed, by definition the projective space over a field 
$\mathbb{F}(\mathbb{F}=\mathbb{C}, \mathbb{H})$ is the set of all the lines through the origin. A natural chart on this manifold is given by the formula:

$$
q_{i}^{(k)}=v_{i} v_{k}^{-1}, \quad i, k=1, \ldots, n+1,
$$

where $v$ define coordinates of the corresponding $\mathbb{F}^{n+1}$ and $q_{i}^{(k)}$ is the $i$-th coordinate of the $k$-th chart of the $\mathbb{F} P^{n}$. This maps define two infinite families of tautological fibrations

$$
S^{2^{N}-1} \rightarrow S^{2^{N}(n+1)-1} \rightarrow \mathbb{F} P^{n}
$$

where $2^{N}(N=1,2)$ is the dimensionality of the corresponding field $\mathbb{F}$. Each first element of these families is the famous Hopf fibration of sphere over sphere:

$$
S^{1} \rightarrow S^{3} \rightarrow S^{2}, \quad S^{3} \rightarrow S^{7} \rightarrow S^{4} .
$$

In our research we are interested in the projectivization of the second fibrations in (2.2). Namely, it is possible to project the total space and the fiber of (2.2) with $N=2$ using the projection of the corresponding fibration with $N=1$ as a map, so that it will not affect the base. After projectivization we will arrive to the following fiber bundle:

$$
S^{2} \rightarrow \mathbb{C} P^{2 n+1} \rightarrow \mathbb{H} P^{n}
$$

Now, let us pass to the explicit construction of these fibrations. We start from the $2 n+2$-dimensional complex plane $\mathbb{C}^{2 n+2} \simeq \mathbb{H}^{n+1}$ with complex coordinates $\lambda$ or quaternionic ones: $v_{i}=\lambda_{2 i-1}+j \lambda_{2 i}($ $i=1, \ldots, n+1)$. By definition the coordinates

$$
q_{\alpha}=v_{\alpha} v_{n+1}^{-1} \equiv v_{\alpha} \frac{\bar{v}_{n}}{\left\|v_{n}\right\|^{2}}, \quad \alpha=1, \ldots, n .
$$

define a chart on the quaternionic projective space $\mathbb{H} P^{n}$.

The inverse formulas look as follows:

$$
v_{\alpha}=q_{\alpha} v_{n+1}=q_{\alpha}\left(\lambda_{2 n+1}+j \lambda_{2 n+2}\right)=\lambda_{2 \alpha-1}+j \lambda_{2 \alpha}
$$

Multiplying the last equation by $\lambda_{2 n+2}^{-1}$ one finds

$$
q_{\alpha}\left(z_{2 n+1}+j\right)=z_{2 \alpha-1}+j z_{2 \alpha} .
$$

where the quantities $z_{r}=\lambda_{r} / \lambda_{2 n+2}(r=1, \ldots, 2 n+1)$ define a chart on the complex projective space $\mathbb{C} P^{2 n+1}$. It is clear, that any coordinate of $\mathbb{C} P^{2 n+1}$ by itself defines a chart on a $\mathbb{C} P^{1} \simeq S^{2}$. In particular, one can consider as such the last coordinate $z_{2 n+1}$.

We can rewrite (2.7) in the following form:

$$
z_{2 \alpha-1}+j z_{2 \alpha}=q_{\alpha}(u+j), \quad z_{2 n+1}=u .
$$

In this form those relations define a natural projection of the fibration (2.4)

The form of transition functions can be easily found from the construction described above.

For our further consideration it is convenient, instead of the quaternionic coordinates $q$, to use complex coordinates $w$ which we introduce by the following formula:

$$
q_{\alpha}=w_{2 \alpha-1}+j w_{2 \alpha}
$$

In these coordinates (2.8) takes the following form:

$$
z_{2 \alpha-1}=w_{2 \alpha-1} u-\bar{w}_{2 \alpha}, \quad z_{2_{\alpha}}=w_{2 \alpha} u+\bar{w}_{2 \alpha-1}, \quad z_{2 n+1}=u .
$$

In order to unify the first two expressions we introduce a matrix $\Omega$ by the following formula:

$$
\left(\Omega_{\mu \nu}\right)=\left(\begin{array}{ccccc}
\varepsilon & 0 & 0 & 0 & \ldots \\
0 & \varepsilon & 0 & 0 & \ldots \\
0 & 0 & \varepsilon & 0 & \ldots \\
\cdots & \ldots & \ldots & \ldots & \ldots
\end{array}\right), \quad \varepsilon=\left(\begin{array}{cc}
0 & 1 \\
-1 & 0
\end{array}\right) .
$$


With this matrix we can rewrite (2.10) in the following form:

$$
z^{\mu}=u w^{\mu}+\Omega^{\mu \nu} \bar{w}_{\nu}, \quad z_{2 n+1}=z^{2 n+1}=u \quad \mu, \nu=1, \ldots, 2 n .
$$

Remark. From this point we will make a difference between the upper and lower indices. We define $w^{\mu}$ with upper index, while its complex conjugate has a lower one: $\bar{w}_{\mu}$. The rule of raising and lowering the indices is given via the matrix $\Omega_{\mu \nu}$ and its inverse $\Omega^{\mu \nu}$ :

$$
\Omega_{\mu \nu} \Omega^{\nu \lambda}=\delta_{\mu}^{\lambda}
$$

Thus, we define

$$
w_{\mu}=\Omega_{\mu \nu} w^{\nu}, \quad \bar{w}^{\mu}=\Omega^{\mu \nu} \bar{w}_{\nu}
$$

The contraction is done, as usual, between upper and lower indices. So,

$$
z \bar{z}=z^{\mu} \bar{z}_{\mu}=-z_{\mu} \bar{z}^{\mu}
$$

Now, using the above established relations between inhomogeneous coordinates of complex and quaternionic spaces, let us relate metrics on these spaces.

It is known that the natural metric on $S^{2^{N}(n+1)-1}$ induces the Fubini-Study metric on the corresponding projective space:

$$
d s^{2}=\frac{d z \bar{d} z}{1+z \bar{z}}-\frac{(\bar{z} d z)(z d \bar{z})}{(1+z \bar{z})^{2}} .
$$

The non-degenerate transformation (2.12) defines a connection on the fibration (2.4). Indeed, replacing the coordinates $z_{i}$ with $(q, u)$ transforms the Fubini-Study metric on $\mathbb{C} P^{2 n+1}$ as follows:

$$
d s^{2}=\frac{d q \bar{d} q}{1+q \bar{q}}-\frac{(\bar{q} d q)(d \bar{q} q)}{(1+q \bar{q})^{2}}+\frac{(d u+A)(d \bar{u}+\bar{A})}{(1+u \bar{u})^{2}},
$$

where

$$
A=\left.j \frac{(\bar{u}-j) \bar{q} d q(u+j)}{1+w \bar{w}}\right|_{\mathbb{C}} \equiv \frac{u(\bar{w} d w-w d \bar{w})-(\bar{w} \Omega d \bar{w})-u^{2}(w \Omega d w)}{1+w \bar{w}} .
$$

Here $\left.q\right|_{\mathbb{C}} \equiv 1 / 2(q-\imath q \imath)$ denotes the complex part of the quaternion $q$. In complex coordinates $w$ the metrics of $\mathbb{H} P^{n}$ reads

$$
g_{\mu}{ }^{\nu}=\frac{\delta_{\mu}^{\nu}}{1+w \bar{w}}-\frac{\bar{w}_{\mu} w^{\nu}+w_{\mu} \bar{w}^{\nu}}{(1+w \bar{w})^{2}} .
$$

The complex projective space is a Riemannian symmetric space. Indeed, each sphere of the total space in (2.4) can be represented as a coset space

$$
U(n+1) / U(n) \simeq S^{2 n+1},
$$

Reducing this by the global factor $U(1)$, we find:

$$
S U(n+1) / U(n) \simeq \mathbb{C} P^{2 n+1} .
$$

Thus, the isometries of the complex projective space form the $s u(n+1)$ algebra. These isometries are defined, in the given parametrization, by the following vector fields

$$
R_{i}=\partial_{i}+\bar{z}_{i}(\bar{z} \bar{\partial}), \quad J_{i}{ }^{j}=\imath\left(z^{j} \partial_{i}-\bar{z}_{i} \bar{\partial}^{j}\right)+\imath \delta_{i}^{j}((z \partial)-(\bar{z} \bar{\partial})) .
$$

These are all we need to know about the transformation of coordinates of the $\mathbb{C} P^{n}$.

Now, we are ready to construct a mechanical system of a particle on $\mathbb{H} P^{n}$ in the vector-potential (2.18) by the reduction of the free particle on $\mathbb{C} P^{2 n+1}$. 


\section{$3 \quad$ Landau problem on $\mathbb{H} P^{n}$ from a free particle on $\mathbb{C} P^{2 n+1}$}

Let us show, that the free particle on $\mathbb{C} P^{2 n+1}$ is immediately reduced to the particle on $\mathbb{H} P^{n}$ in the presence of BPST instanton field (which is natural to call the "Landau problem on $\mathbb{H} P^{n}$ ").

In accordance with (2.16), the free particle on $\mathbb{C} P^{2 n+1}$ is defined by the Lagrangian

$$
L_{0}=\frac{\dot{\bar{z}} \cdot \dot{z}}{1+z \bar{z}}-\frac{(\dot{\bar{z}} z)(\dot{z} \bar{z})}{(1+z \bar{z})^{2}}
$$

In terms of (2.12) it reads

$$
L=\frac{\dot{q} \dot{\bar{q}}}{1+q \bar{q}}-\frac{(\bar{q} \dot{q})(\dot{\bar{q}} q)}{(1+q \bar{q})^{2}}+\frac{(\dot{u}+A)(\dot{\bar{u}}+\bar{A})}{(1+u \bar{u})^{2}} .
$$

In order to reduce it to the system on $\mathbb{H} P^{n}$, it is convenient to give the Hamiltonian formulation of this system and then perform the Hamiltonian reduction associated with the Hopf map. Precisely, in the Hamiltonian language the free particle system on $\mathbb{C} P^{n}$ is defined by the triple

$$
\left(H_{0}=\left(g^{-1}\right)_{i}{ }^{j} p_{i} \bar{p}^{j}, \quad \omega=d p_{i} \wedge d z^{i}+d \bar{p}_{i} \wedge d \bar{z}^{i}, \quad T^{*} \mathbb{C} P^{2 n+1}\right),
$$

where $\left(g^{-1}\right)_{i}{ }^{j}=(1+z \bar{z})\left(\delta_{i}^{j}+\bar{z}_{i} z^{j}\right)$ are the components of the inverse Fubini-Study metric (2.16). This system possesses the $s u(2 n+2)$ symmetry algebra given by the generators (2.22). These generators define the following Noether constants of motion

$$
R_{i}=p_{i}+\bar{z}_{i}(\bar{z} \bar{p}), \quad J_{i}{ }^{j}=\imath\left(z^{j} p_{i}-\bar{z}_{i} \bar{p}^{j}\right)+\imath \delta_{i}^{j}((z p)-(\bar{z} \bar{p})) .
$$

We can extend the transformation (2.12) to the canonical one by adding the following transformation rule for the conjugated momenta:

$$
p_{\mu}=\frac{\bar{u}}{1+u \bar{u}} \pi_{\mu}+\frac{1}{1+u \bar{u}} \bar{\pi}_{\mu}, \quad p_{2 n+1}=p_{u}-\frac{1}{1+u \bar{u}}\left(\bar{u} w^{\mu} \pi_{\mu}-w^{\mu} \bar{\pi}_{\mu}\right) .
$$

It is an exercise to check that the canonical transformation (2.10), (3.5) leads to the following form of the Hamiltonian:

$$
H_{0}=\left(g^{-1}\right)_{\mu}{ }^{\nu} \bar{P}^{\mu} P_{\nu}+(1+u \bar{u})^{2} p_{u} \bar{p}_{u},
$$

where we introduced the inverse metric to (2.19)

$$
\left(g^{-1}\right)_{\mu}^{\nu}=(1+w \bar{w})\left(\delta_{\mu}^{\nu}+\bar{w}_{\mu} w^{\nu}+w_{\mu} \bar{w}^{\nu}\right)
$$

and the covariant momenta

$$
P_{\mu}=\pi_{\mu}-\imath \bar{w}_{\mu} \frac{I_{3}}{1+w \bar{w}}-w_{\mu} \frac{I_{+}}{1+w \bar{w}},
$$

with the $s u(2)$ generators $I_{ \pm}, I_{3}$ defining the isometries of $S^{2}$ :

$$
\begin{gathered}
I_{3}=-\imath\left(u p_{u}-\bar{u} \bar{p}_{u}\right), \quad I_{+}=\bar{p}_{u}+u^{2} p_{u}, \quad I_{-}=p_{u}+\bar{u}^{2} \bar{p}_{u}, \\
\left\{I_{3}, I_{ \pm}\right\}= \pm \imath I_{ \pm}, \quad\left\{I_{+}, I_{-}\right\}=2 \imath I_{3},
\end{gathered}
$$

The Poisson brackets between the quantities $P_{\mu}$ read

$$
\left\{w^{\mu}, P_{\nu}\right\}=\delta_{\nu}^{\mu}, \quad\left\{P_{\mu}, P_{\nu}\right\}=-2 \frac{\Omega_{\mu \nu}}{1+w \bar{w}} I_{+}, \quad\left\{P_{\mu}, \bar{P}^{\nu}\right\}=\imath \frac{\delta_{\mu}^{\nu} I_{3}}{(1+w \bar{w})^{2}} .
$$

Besides, we have

$$
\left\{P_{\mu}, I_{+}\right\}=\frac{\bar{w}_{\mu} I_{+}}{1+w \bar{w}}, \quad\left\{P_{\mu}, I_{-}\right\}=-\frac{\bar{w}_{\mu} I_{-}}{1+w \bar{w}}-2 \imath \frac{w_{\mu} I_{3}}{1+w \bar{w}}, \quad\left\{P_{\mu}, I_{3}\right\}=\frac{\imath w_{\mu} I_{+}}{1+w \bar{w}} .
$$

Let us also present, for completeness, the some other relations as well

$$
\left\{I_{3}, p_{u}\right\}=-\imath p_{u}, \quad\left\{I_{+}, p_{u}\right\}=2 u p_{u}, \quad\left\{I_{-}, p_{u}\right\}=0, \quad\left\{P_{\mu}, p_{u}\right\}=-\frac{p_{u}}{1+w \bar{w}}\left(\bar{w}_{\mu}+2 u w_{\mu}\right)
$$


It is easy to see that the Casimir operator of these $s u(2)$ generators is precisely the Hamiltonian of a free particle moving on $S^{2}$, in (3.6) as a second summand:

$$
I^{2}=I_{+} I_{-}+I_{3}^{2}=(1+u \bar{u})^{2} p_{u} \bar{p}_{u}
$$

Obviously, it commutes with the Hamiltonian and defines an integral of motion of the system.

Our goal is to perform the Hamiltonian reduction by this constant of motion. For this purpose we should fix its $(4(2 n+1)-1)$-dimensional level surface, by putting

$$
I^{2}=s^{2},
$$

and then factorize it by the vector flow $\left\{I^{2},\right\}$ to the $(8 n+2)=(2 \cdot 4 n+2)$ dimensional phase space.

The role of the $8 n$ coordinates of the reduced phase space could be played by $P_{\mu}, w^{\mu}$ given by (3.5), (2.12). To find the rest two coordinates, we should simply resolve the condition (3.14) preserving Poisson brackets (3.10):

$$
I_{+}=s \frac{2 x}{1+x \bar{x}}, \quad I_{3}=s \frac{1-x \bar{x}}{1+x \bar{x}}, \quad s \in \mathbb{R}, \quad x \in \mathbb{C} .
$$

This yields

$$
\begin{gathered}
x=\frac{I_{+}}{s+I_{3}},:\{x, \bar{x}\}=\frac{i}{2 s}(1+x \bar{x})^{2}, \quad\left\{P_{\mu}, x\right\}=\frac{\bar{w}_{\mu} x}{1+w \bar{w}}-\imath \frac{x^{2} \bar{w}_{\mu}}{1+w \bar{w}}, \\
\left\{P_{\mu}, \bar{x}\right\}=-\frac{\bar{w}_{\mu} \bar{x}+\imath w_{\mu}}{1+w \bar{w}}, \quad\left\{w^{\mu}, x\right\}=\left\{w^{\mu}, \bar{x}\right\}=0
\end{gathered}
$$

Thus, we have reduced the phase space $T^{*} \mathbb{C} P^{2 n+1}$ to the $T^{*} \mathbb{H} P^{n} \times S^{2}$. The latter defines the phase space of the (su(2)-)isospin particle on $\mathbb{H} P^{n}$ interacting with the BPST instanton field. Its Poisson brackets are defined by the relations (3.11), (3.17), or, equivalently, by (3.10), (3.11), (3.12).

The Hamiltonian of the free particle on $\mathbb{C} P^{2 n+1}$ given by (3.3) (or, equivalently, by (3.6)), results, upon reduction, to the one on $\mathbb{H} P^{n}$,

$$
H_{\mathbb{H} P^{n}}=\left(g^{-1}\right)_{\mu}{ }^{\nu} \bar{P}^{\mu} P_{\nu}+s^{2} .
$$

So, we reduced the free particle system on $T^{*} \mathbb{C} P^{2 n+1}$ to the isospin particle on $\mathbb{H} P^{n}$ interacting with the BPST instanton field. The subset of the Noether constants of motion (3.4) commuting with (3.14) is reduced on $\mathbb{H} P^{n}$ and form the $S p(n+1)$ algebra of the isometries of the reduced system.

These reduced generators are given by the expressions

$$
\begin{gathered}
L_{\mu}{ }^{\nu}=J_{\mu}{ }^{\nu}+J^{\nu}{ }_{\mu}=\imath\left(w^{\nu} \pi_{\mu}-\bar{w}_{\mu} \bar{\pi}^{\nu}\right)+\imath\left(w_{\mu} \pi^{\nu}-\bar{w}^{\nu} \bar{\pi}_{\mu}\right) \\
L_{3}=J_{2 n+1}{ }^{2 n+1}=I_{3}+\frac{\imath}{2}((\pi w)-(\bar{\pi} \bar{w})), \quad L_{-}=R_{2 n+1}=\left(\bar{\pi}^{\mu} w_{\mu}\right)+I_{-}, \quad L_{+}=\bar{L}_{-} \\
L^{\mu}=\imath J_{2 n+1}{ }^{\mu}-R^{\mu}=\bar{\pi}^{\mu}-\left(\left(\bar{\pi}^{\nu} w_{\nu}\right)-I_{-}\right) \bar{w}^{\mu}+\left((\pi w)-\imath I_{3}\right) w^{\mu}
\end{gathered}
$$

The $2 n(2 n+1) / 2$ generators $L_{\mu \nu}$ and three generators $L_{ \pm}, L_{3}$, form the $s p(n) \times s p(1)$ algebra, and these two sets of generators, together with the $4 n$ generators $L_{\mu}$, form the $2 n^{2}+5 n+3$-dimensional algebra of the isometries of $\mathbb{H} P^{n}$, that is $s p(n+1)$ :

$$
\begin{gathered}
\left\{L_{\mu \nu}, L_{\rho \sigma}\right\}=-\imath\left(\Omega_{\mu \rho} L_{\sigma \nu}+\Omega_{\nu \rho} L_{\sigma \mu}+\Omega_{\nu \sigma} L_{\mu \rho}+\Omega_{\mu \sigma} L_{\nu \rho}\right) \\
\left\{L_{+}, L_{-}\right\}=2 \imath L_{3}, \quad\left\{L_{3}, L_{ \pm}\right\}= \pm \imath L_{ \pm}, \quad\left\{L_{\mu \nu}, L_{ \pm}\right\}=\left\{L_{\mu \nu}, L_{3}\right\}=0 \\
\left\{L^{\mu}, L_{-}\right\}=0, \quad\left\{L^{\mu}, L_{+}\right\}=\bar{L}^{\mu}, \quad\left\{L^{\mu}, L_{3}\right\}=\frac{\imath}{2} L^{\mu}, \quad\left\{L^{\mu}, L_{\rho}{ }^{\sigma}\right\}=-\imath \delta_{\rho}^{\mu} L^{\sigma}-\imath L_{\rho} \Omega^{\mu \sigma} \\
\left\{L^{\mu}, L^{\nu}\right\}=2 \Omega^{\mu \nu} L_{-}, \quad\left\{\bar{L}_{\mu}, \bar{L}_{\nu}\right\}=-2 \Omega_{\mu \nu} L_{+}, \quad\left\{L^{\mu}, \bar{L}_{\nu}\right\}=\imath L_{\nu}{ }^{\mu}+2 \imath \delta_{\nu}^{\mu} L_{3}
\end{gathered}
$$

The Casimir of $s p(n+1)$ is precisely the Hamiltonian on $\mathbb{H} P^{n}$

$$
H_{\mathbb{H} P^{n}}=\frac{L_{\mu}{ }^{\nu} L_{\nu}{ }^{\mu}+4 L^{\mu} \bar{L}_{\mu}+8\left(L_{+} L_{-}+L_{3}^{2}\right)}{4}+I^{2}
$$

Hence, we established a complete correspondence between the $S U(2)$ (classical) Landau problem on $\mathbb{H} P^{n}$ and the free particle system on $\mathbb{C} P^{2 n+2}$ 


\section{Oscillator}

In the previous section we considered the free particle on the n-dimensional quaternionic projective space $\mathbb{H} P^{n}$ and demonstrated, that the inclusion of $S U(2)$ instanton preserves its whole symmetry algebra $s p(n+1)$. In fact $\mathbb{H} P^{n}$ seems to be a natural candidate on the role of configuration spaces of the (super)integrable systems interacting with the BPST instanton field. At least, on these spaces there should exist the proper generalizations of the systems on $\mathbb{R}^{4}$ respecting the inclusion of the BPST instanton. The simplest system of this sort, besides the free particle, is the $4 n$-dimensional isotropic oscillator. How to construct its appropriate analog on $\mathbb{H} P^{n}$ ?

Let us consider a more complicated integrable system on $\mathbb{H} P^{n}$, that is the generalization of oscillator, given by the following expression:

$$
H_{o s c}=H_{\mathbb{H} P^{n}}+\omega_{0}^{2} w \bar{w},
$$

where the first term is simply the free particle Hamiltonian on $\mathbb{H} P^{n}$ given by (3.18).

This potential has been suggested in [10, 11] in analogy with the earlier constructed oscillator potential on $\mathbb{C} P^{n}$. In our opinion, it is deductive to present here the speculations which lead to the suggestion of above system. Namely, in [7 two of the authors constructed the model of the oscillator on $\mathbb{C} P^{n}$ requiring that it should have the hidden symmetries, resulting, in the flat limit, in the ordinary oscillator on $\mathbb{C}^{n}$. Such a model was found to be unique. On $\mathbb{C} P^{1}$ it was found to be the well-known Higgs oscillator, while for $n>1$ it was defined by the potential

$$
V_{\mathbb{C} P^{n}}=\omega_{0}^{2} z \bar{z},
$$

with $z^{i}$ being the inhomogeneous coordinates on $\mathbb{C} P^{n}$. Besides the $u(n)$ Nöther constants of motion defined by the second expression in (3.4), this system was found to have hidden constants of motion (for $n>1$ ) given by the expression

$$
I_{j}^{i}=\bar{R}^{i} R_{j}+\omega_{0}^{2} z^{i} \bar{z}_{j}
$$

where $R_{i}$ are the translation generators defined by the first expression in (3.4) Surprisingly, it was found that the inclusion of of the constant magnetic field preserves all symmetries (and, respectively, superintegrability) of the system! Moreover, it was found, that even on $\mathbb{C} P^{1}$ this potential is a distinguished one. Namely, though the system is not superintegrable in this case, it is exactly solvable, and preserves the exact solvability property after inclusion of the constant magnetic field, while the Higgs oscillator on

$S^{2}=\mathbb{C} P^{1}$, being a superintegrable system, looses the superintegrability (and even the exact solvability) property upon inclusion of a constant magnetic field. This allowed the authors to call that system "C $P^{n}$-oscillator" for any $n$. It was further studied in 8 .

Taking in mind, that the potential of the Higgs oscillator on the n-dimensional sphere (to be more precise, on the real projective space) reads, in inhomogeneous coordinates

$$
V_{\mathbb{R} P n}=\frac{\omega_{0}^{2} y^{2}}{2}, \quad y^{i}=\frac{u^{i}}{u^{0}}
$$

with $u^{i}, u^{0}$ being coordinates of the ambient $\mathbb{R}^{n}$ space $\left(\left(u^{i}\right)^{2}+\left(u^{0}\right)^{2}=1\right)$, the authors of 10, 11] claimed, that the oscillator potential on $\mathbb{H} P^{n}$ should be given by the same expression, as in the case of $\mathbb{C} P^{n}$, with the replacement of inhomogeneous complex coordinates with quaternionic ones. And this system has to respect the inclusion of the BPST instanton field. They have checked this in the simplest case of $\mathbb{H} P^{n}=S^{4}$ and found that it is indeed the case. However, in contrast with the Higgs oscillator on $\mathbb{R} P^{1}=S^{1}$, and with the $\mathbb{C} P^{1}\left(=S^{2}\right)$-oscillator, the spectrum of the $\mathbb{H} P^{1}$-oscillator (and of its hyperbolic analog [11]) system was found to be degenerated, which is a precise indication of the existence of hidden symmetries. Unfortunately, no explanation of these symmetries has been done there. Moreover, this claim has never been checked for nontrivial (higher-dimensional) cases.

Now, let us show, that the Hamiltonian (4.1), together with Poisson brackets (3.10)-(3.12) defines a well-defined oscillator system on $\mathbb{H} P^{n}$.

It is clear that the added oscillator potential does not commute with the coset generators $L^{\mu}, \bar{L}_{\mu}$, while the rest of the $s p(n) \times s p(1)$ generators $L_{\mu}{ }^{\nu}, L_{ \pm, 3}$ remain as symmetries of the system. However, the system possesses a set of hidden symmetries:

$$
I_{\nu}^{\mu}=L^{\mu} \bar{L}_{\nu}-\bar{L}^{\mu} L_{\nu}+\omega_{0}^{2}\left(w^{\mu} \bar{w}_{\nu}-\bar{w}^{\mu} w_{\nu}\right)
$$


which are constructed by analogy with the corresponding integrals for the $\mathbb{C} P^{n}$ oscillator (4.3). These quantities commute with $L_{ \pm, 3}$ and transform linearly with respect to $L_{\mu \nu}$ :

$$
\left\{I_{\mu \nu}, L_{\rho \sigma}\right\}=\imath\left(\Omega_{\mu \sigma} I_{\nu \rho}+\Omega_{\rho \mu} I_{\sigma \nu}-\Omega_{\nu \sigma} I_{\mu \rho}-\Omega_{\nu \rho} I_{\sigma \mu}\right), \quad\left\{L_{\mu \nu}, L_{ \pm, 3}\right\}=\left\{I_{\mu \nu}, L_{ \pm, 3}\right\}=0,
$$

However, in contrast to the case of the $\mathbb{C} P^{n}$ oscillator, where the symmetries of the system form a quadratic algebra, in the case of the $\mathbb{H} P^{n}$ oscillator the Poisson brackets between the hidden symmetry generators cannot be expressed through the combination of $L$ and $I$ and give us a new set of integrals of motion, which are, already, cubic in momenta:

$$
\left\{I_{\mu \nu}, I_{\rho \sigma}\right\}=\imath\left(I_{\mu \rho} L_{\nu \sigma}+I_{\nu \sigma} L_{\mu \rho}-I_{\mu \sigma} L_{\nu \rho}-I_{\nu \rho} L_{\mu \sigma}\right)+\Omega_{\mu \rho} S_{\nu \sigma}+\Omega_{\nu \sigma} S_{\mu \rho}-\Omega_{\mu \sigma} S_{\nu \rho}-\Omega_{\nu \rho} S_{\mu \sigma}
$$

where

$$
\begin{gathered}
S_{\mu \nu}=2 L_{\mu} L_{\nu} L_{-}+2 \bar{L}_{\mu} \bar{L}_{\nu} L_{+}+2 \imath L_{3}\left(L_{\mu} \bar{L}_{\nu}+L_{\nu} \bar{L}_{\mu}\right)- \\
-\omega_{0}^{2}\left(L_{\mu \nu}+2\left(w_{\mu} w_{\nu} L_{+}+\bar{w}_{\mu} \bar{w}_{\nu} L_{-}-\imath L_{3}\left(w_{\mu} \bar{w}_{\nu}+w_{\nu} \bar{w}_{\mu}\right)\right)\right)
\end{gathered}
$$

defines the new set of cubic constants of motion. Their Poisson brackets yield additional, last, set of constants of motion

$$
T_{\mu \nu}=I_{-}\left(\bar{\pi}_{\mu} w_{\nu}-\bar{\pi}_{\nu} w_{\mu}\right)+I_{+}\left(\pi_{\mu} \bar{w}_{\nu}-\pi_{\nu} \bar{w}_{\mu}\right)-\imath I_{3}\left(\pi_{\mu} w_{\nu}-\pi_{\nu} w_{\mu}+\bar{\pi}_{\mu} \bar{w}_{\nu}-\bar{\pi}_{\nu} \bar{w}_{\mu}\right) .
$$

Let us notice, that the constants of motion (4.8), (4.9) have no analogs neither in Higgs, no in $\mathbb{C} P^{n}$ oscillator models. It seems, that precisely these constants of motion are responsible for the degeneracy of the spectrum of the $\mathbb{H} P^{1}$ oscillator observed in [11].

Finally, let us notice, that repeating the speculations given for the $\mathbb{C} P^{n}$ oscillator, we can define the singular version of the $\mathbb{H} P^{n}$ oscillator respecting the inclusion of the BPST instanton field,

$$
V=\frac{\alpha^{2}}{w \bar{w}}+\omega_{0}^{2} w \bar{w} .
$$

The classical and semiclassical analysis of its $\mathbb{C} P^{1}$ analog has been done in [12.

\section{Conclusion}

In this paper we constructed two basic one-particle integrable systems on quaternionic projective spaces, that are the "quaternionic Landau problem" on $\mathbb{H} P^{n}$, i.e. the particle moving in the presence of an instanton field, and the " $\mathbb{H} P^{n}$-oscillator" (interacting with the instanton field). Both systems are superintegrable: the first one possesses the $S p(n+1)$ symmetry algebra, while the symmetry algebra of the second one is highly nonlinear, and it still needs to be calculated. Note, that both systems can be easily lifted to the ones on the complex projective space, where the instanton field is "absorbed" in the spatial coordinates. The obvious next step is to consider the respective quantum mechanical systems for $n>1$ (for the $n=1$ case it was considered in earlier works). With the quantum mechanics at hands, one can consider, e.g. the "quantum Hall effect on $\mathbb{H} P^{n}$ ", in analogy with "quantum Hall effect on $\mathbb{C} P^{n}$ ", considered by Karabali and Nair (see the fourth reference in [4] and [5]). A more detailed description of the "singular $\mathbb{H} P^{n}$-oscillator" defined by the potential (4.10), and the construction and proper generalization of the Coulomb system are also in order. Supersymmetric extensions of these systems, which are similar to those on complex projective spaces, are of special importance [13. However, the natural desire to obtain them by the Hamiltonian reduction from the complex projective space seems to be technically irrelevant [14, so that one should try to do it in a less obvious way.

Acknowledgements. We thank Tigran Hakobyan and David Karakhanyan for useful comments. This work was supported by the Armenian State Committee of Science grant 11-1c258, by the ANSEF grant 2908, by RFBR grants 11-02-01335, 12-02-00517, and 11-02-90445-Ukr as well as by ERC Advanced Grant no. 226455, Supersymmetry, Quantum Gravity and Gauge Fields (SUPERFIELDS). 


\section{References}

[1] M. F. Atiah, Geometry of the Yang-Mills Fields, Accademia Nazionale dei Lincei, Scuola Normale Superiore, Lezioni Ferminale, Pisa, 1979.

[2] C. Duval and P. Horvathy, Annals Phys. 142, 10 (1982).

[3] S. -C. Zhang and J. -p. Hu, Science 294 (2001) 823 cond-mat/0110572.

J. P. Hu, S. C. Zhang, Phys. Rev. B 66 (2002), 125301 cond-mat/0112432.

[4] B. A. Bernevig, C. H. Chern, J. P. Hu, N. Toumbas, S. C. Zhang, Annals Phys. 300 (2002) 185.

M. Fabinger, JHEP 0205 (2002) 037.

Y. X. Chen, B. Y. Hou, B. Y. Hou, Nucl. Phys. B638 (2002) 220.

D. Karabali, V. P. Nair,Nucl. Phys.B641(2002) 533.

A. P. Polychronakos, Nucl.Phys.B705 (2005) 457 hep-th/0408194; Nucl.Phys.B711(2005),505 hep-th/0411065.

[5] D. Karabali and V. P. Nair, J. Phys. A 39, 12735 (2006) hep-th/0606161.

[6] S. Bellucci, P. -Y. Casteill and A. Nersessian, Phys. Lett. B 574, 121 (2003) hep-th/0306277.

[7] S. Bellucci and A. Nersessian, Phys. Rev. D 67, 065013 (2003) [Erratum-ibid. D 71, 089901 (2005)] hep-th/0211070.

[8] S. Bellucci, A. Nersessian and A. Yeranyan, Phys. Rev. D 70 (2004) 085013 [hep-th/0406184]; S. Bellucci, A. Nersessian and A. Yeranyan, Phys. Rev. D 70, 045006 (2004) hep-th/0312323.

[9] P. W. Higgs J. Phys. A: Math. Gen. 12309 (1979);

H. I. Leemon, J. Phys. A 12 (1979) 489.

[10] A. Nersessian, Lect. Notes Phys. 698 (2006) 139 [hep-th/0506170].

[11] L. Mardoyan and A. Nersessian, Phys. Rev. B 72 (2005) 233303 hep-th/0508062.

S. Bellucci, L. Mardoyan and A. Nersessian, Phys. Lett. B 636 (2006) 137 hep-th/0602231.

[12] K. S. Aramyan, Teor. Mat. Fiz. 156 (2008) 131.

S. Bellucci, A. Nersessian, A. Saghatelian, V. Yeghikyan, J. Comput. Theor. Nanosci. 8 (2011) 769 arXiv:1008.3865.

[13] S. Bellucci, A. Nersessian, Phys. Rev. D 64 (2001) 021702 arXiv:hep-th/0101065.

S. Bellucci, S. Krivonos and A. Sutulin, Phys. Rev. D 81(2010) 105026.

S. Bellucci, N. Kozyrev, S. Krivonos and A. Sutulin, "Symmetries of N=4 supersymmetric CP(n) mechanics," arXiv:1206.0175 [hep-th]. S. Bellucci, A. Nersessian, Nucl. Phys. Proc. Suppl. 102 (2001) 227, arXiv:hep-th/0103005

[14] N. Kozyrev, S. Krivonos and O. Lechtenfeld, "N=2 supersymmetric $S^{2} \rightarrow C P^{3} \rightarrow S^{4}$ fibration viewed as superparticle mechanics," arXiv:1210.4587 [hep-th]. 